\title{
THE ROLE OF INTERVENTIONAL RADIOLOGY IN MODERN MEDICAL PRACTICE.
}

\author{
*A.O Akhigbe \\ *Department of Radiology, University of Benin Teaching Hospital Benin City. \\ Correspondence: \\ Dr A.O Akhigbe \\ Department of Radiology, \\ University of Benin Teaching Hospital \\ P.M.B. 1111 Benin City \\ Edo State, Nigeria.
}

\begin{abstract}
WHAT IS INTERVENTIONAL RADIOLOGY (IR)? This is a subspecialty in diagnostic Radiology, which involves the use of minimally invasive targeted treatment options to manage various medical conditions, under imaging guidance.

This treatment method is less invasive compared with surgery and most times does not require general anesthesia. They are most often done as outpatient treatment with reduced morbidity, mortality and shorter off duty period.
\end{abstract}

\section{AVAILABLE IR PROCEDURES AND APPLICATIONS \\ 1. BALLOON ANGIOPLASTY AND STENTING \\ ANGIOPLASTY}

This is an IR procedure involving the use of balloon to dilate areas of stenosis in arteries and veins.

The area of stenosis is assessed using catheter and guide wires and appropriate sized balloon is inflated at the site of stenosis to widen the area of narrowing and the balloon is removed after the procedure.

\section{STENTING}

In some cases where the wall of the area of stenosis is compromised and cannot be maintained with just angioplasty, a stent is put across the area of stenosis, using either the balloon expandable or self-expanding type of stent.

\section{APPLICATION:}

PERIPHERAL ARTERIAL DISEASE (PAD)

Patients presenting with intermittent claudication, rest pain, ischaemic ulceration and gangrene as complications of DM, hypertension, atherosclerosis.

This can be done as alternatives to reconstructive vascular surgery.

Particularly useful to prevent amputation in pts with rest pain, ulceration and gangrene that are unfit for surgery (limb salvage angioplasty). The need for routine screening for PAD in Diabetes Melitus and Hypertension using the pulse Volume Recording (PVR) and Ankle Brachial Index (ABI) is highly recommended for early diagnosis of PAD when the limb can still be salvaged.

\section{CORONARY ARTERIAL DISEASE}

Patient presenting with myocardial infarction are treated with angioplasty and stenting of the affected coronary artery and this is often done as out patient procedure.

Other cardiac interventions using this same IR procedures include; balloon valvuloplasty of the pulmonary, aortic, 
mitral and tricuspid valves. Balloon dilatation of aortic coarctation and closure of PDA.

\section{RENAL ANGIOPLASTY}

This is an interventional procedure as treatment option in renovascular hypertension.

Reported success rate of $90 \%$ for stenosis and $50 \%$ for occlusion..

Long-term results show that $95 \%$ of pts with fibromuscular dysplasia benefit from percutaneous transluminal angioplasty (PTA) with $60 \%$ cure of their hypertension and $35 \%$ improved blood pressure control.

\section{CAROTID ANGIOPLASTY}

Innominate, carotid and vertebral artery stenoses from plaques or atheromas presenting with TIA can be treated by PTA or stents.

This is an alternative to endarterectomy.

\section{ABDOMINAL AORTIC ANEURYSM}

Interventional treatment is possible using a stent and graft.

This is done using imaging to guide catheter and graft through the femoral artery with a small incision at the groin. This is in comparison with surgical repair which will require a large laparotomy abdominal incision, clamping of the aorta and longer hospital admission, with higher mortality rate.

\section{MESENTERIC ANGIOPLASTY}

For repair of celiac and superior mesenteric artery stenosis and occlusion in pts with chronic mesenteric ischaemia.

\section{Other available adjunctive} techniques to angioplasty include;

- Mechanical rotating devices for recanalization of complete vascular occlusion.

- Atheroma removing device.

- Laser assisted angioplasty.
VENOUS STENTS

Used for management of patients with SVC obstruction secondary to bronchial carcinoma or other mediastinal tumours

\section{INFERIOR VENA CAVAL} FILTERS.

There are temporary and permanent filters which can be inserted into the IVC to reduce the rate of recurrent pulmonary emboli in patients with predisposing conditions.

3. OTHER PROCEDURES UNDER VASCULAR INTERVENTION INCLUDE;

- Transjugular intrahepatic portosystemic shunt (TIPS)

This involves creation of an artificial pathway for blood to flow from the main portal vein directly to the hepatic veins, bypassing the venules of the liver.

Indications include; recurrent GI bleeding in pts with varices despite endoscopic sclerotherapy, intractable ascites in pts with chronic liver disease and Budd-Chiari syndrome.

- Central venous catheters

- Retrieval of vascular foreign bodies.

\section{THROMBOLYSIS}

This involves the direct use of fibrinogens to dissolve blood clot within vessels under ultrasound guidance APPLICATION

- Direct fibrinolytic therapy; Used to treat thrombus within arteries.

- DVT to prevent secondary damage to the venous valves and pulmonary embolism.

This has been found to be more effective and has reduced risk of haemorrhagic complications compared with systemic fibrinolytic agents.

5. EMBOLISATION; This involves the injection of embolic material through a catheter selectively positioned in an artery or vein in other to occlude the artery, vein or vascular bed of an organ or tumour. 
Commonly used embolic materials include; gelfoam, polyvinyl alcohol particles, spiral metal coils, ethanol and detachable balloons etc.

Most of these are used for permanent vascular occlusion but gelfoam is used for temporary occlusion.

\section{APPLICATIONS}

- Therapeutic embolisation; uses include acute haemorrhage, tumour embolisation, (especially vascular tumours), aneurysms, AVMs.

- Selective chemoembolization; delivering drugs directly to site of lesion, eg chemotherapy in malignancy.

- Organ or tumour embolisation e.g adrenal and parathyroid adenomas, hepatic lesions, the spleen in hypersplenism and priapism (gelfoam embolisation of the internal pudendal artery)

- Fibroid embolisation.

\section{INTERVENTIONAL RADIOLOGY TREATMENTS FOR INFERTILITY.}

Some caused of infertility in both men and women can be treated with interventional radiology without hospitalization or general anesthesia.

FEMALE; Commonest cause of infertility is tubal blockage; Diagnosis is usually made with HSG and image guided selective salpingography can be used to open the blocked tube. This has been found to be effective in cornual tubal blockage.

MALE; Varicoceles affecting the testicle and scrotum is a known cause of infertility.

Varicocele embolization is a nonsurgical treatment which is as effective as surgery (ligation), with less risk, less pain and shorter recovery time.

\section{THERMAL ABLATION} TREATMENTS; This involves the use of various forms of energy to kill tissues and make them shrink.

\section{TYPES;}

RADIOFREQUENCY (RF) ABLATION

- This is a non-surgical treatment option for cancerous tumors.

A small needle is inserted through the skin into the tumor and RF energy is transmitted into the target tissue where it produces heat and kills the tumor cells.

This is effective for small to medium size tumors and ideal for patients who are not fit for surgery.

Being a local treatment which does not harm healthy tissue, this procedure can be repeated as often as needed for the comfort of the patient.

It's a safe procedure with few complications.

Ablation can be combined with surgery to ensure all malignant cells are removed.

Applications include; localized liver tumors, breast cancer in elderly women,

Benefits of RF ablation

- Low complication rate

- May be done under conscious sedation or GA

- Well tolerated. Most patients can resume their normal routines next day.

- Can be separated as necessary.

- May be combined with other treatment options.

- Can relieve pain, obstruction and sufferings for many cancer patients (especially in our setting where many are afraid of operation)

- CRYOABLATION

Similar to RFA in that this is also delivered directly into the tumor by a probe inserted through the skin.

This procedure uses extremely cold gas to kill the tumor cell by freezing. 
Initially used for many years intraoperatively by surgeons but with reduction in the size of available needles, the procedure is possible through a small skin nick.

\section{- LASER THERAPY}

This is done under image guidance with insertion of laser needle which causes localized tumor cell death.

- HIGH FREQUENCY FOCUSED ULTRASOUND ABLATION

This is a new treatment option being used for fibroid ablation under MRI guidance.

Very high frequency ultrasound is used to ablate fibroid masses.

\section{IMAGE GUIDED BIOPSY}

Guidance can be by US, CT, MRI, FLUOROSCOPY, MAMMOGRAPHY AND STEREOTACTIC EQUIPMENTS. Application; can be applied to almost all organs in the body.
9. IMAGE GUIDED DRAINAGE

Application include; breast cysts, severe hydronephrosis using percutaneous route, distended gall bladder with obstruction, pelvic abscess collection etc

NEW CANCER TREATMENTS ON THE HORIZON

- MAGNETIC CHEMOTHERAPY

- GENE THERAPY

\section{REFERENCES}

1. Text book of Radiology and Imaging by David Sutton, $7^{\text {th }}$ edition, Churchill Livingstone 2003.

2. The complete guide to vascular ultrasound by Arger $\mathrm{PH}$ \& lyoob SD; Lippincott, Williams \& Wilkins 2004.

This article was a lecture delivered at the Association of Resident Doctor, University of Benin Teaching, Benin City Annual General Meeting/Scientific Conference 2008. 